\title{
Surgery of Upper Basilar Artery Aneurysm
}

\author{
Kentaro Mori, Terushige Toyooka, and Naoki Otani
}

\subsection{Signs and Symptoms}

Aneurysms of the basilar artery (BA) may occur at several locations including the BA-vertebral artery junction, BA-anterior inferior cerebellar artery junction, BA-superior cerebellar artery junction, and BA tip. Upper BA aneurysm is usually considered to include BA tip aneurysm and BA-superior cerebellar artery junction aneurysm. BA aneurysm seldom manifests as neurological problems before rupture. Partially thrombosed BA aneurysm may manifest as progressive neurological deficits due to compression of the brain stem and cranial nerves caused by aneurysm growth. Unruptured BA tip aneurysm has a higher tendency to rupture compared to other types of cerebral aneurysm. Therefore, the surgical indications for unruptured BA aneurysm should consider this fact as well as the age, size, and shape of the aneurysm.

\subsection{Investigation}

Upper BA aneurysms are surrounded by many important osseous and neurovascular structures such as the anterior (ACP) and posterior

K. Mori $(\bowtie) \cdot$ T. Toyooka $\cdot$ N. Otani

Department of Neurosurgery, National Defense Medical College, Tokorozawa, Saitama, Japan e-mail: kmori@ndmc.ac.jp; toyo@ndmc.ac.jp; naotani@ndmc.ac.jp clinoid processes (PCP), optic nerve, oculomotor nerve, internal carotid artery (ICA), posterior communicating artery and its perforators (anterior thalamoperforating arteries), and posterior cerebral artery and its perforators (posterior thalamoperforating arteries: PTPAs) (Fig. 17.1). The PTPAs arise from the superior or posterosuperior surface of the P1 segment of the posterior cerebral artery and at a distance of $0.41-4.71 \mathrm{~mm}$ (mean $1.93 \mathrm{~mm}$ ) from the BA apex. The PTPAs number 1-8 (mean 3.6) with diameter of $0.24-1.18 \mathrm{~mm}$ (mean $0.7 \mathrm{~mm}$ ) [1]. Dissection of the PTPAs from the BA tip aneurysm is the key step to successful clipping surgery.

\subsection{Preoperative Preparation}

Three-dimensional computed tomography angiography and/or digital subtraction angiography are mandatory to investigate the BA complex with the aneurysm (Fig. 17.2). The distance of the aneurysm neck from the clinoid line (the line between the tips of the ACP and PCP) is particularly important to determine the operative approach. Anterolateral approaches such as the pterional transsylvian approach and extradural temporopolar approach (ETA) are suitable for the treatment of BA aneurysms located between $15 \mathrm{~mm}$ above and $5 \mathrm{~mm}$ below the clinoid line. The subtemporal transtentorial approach or 


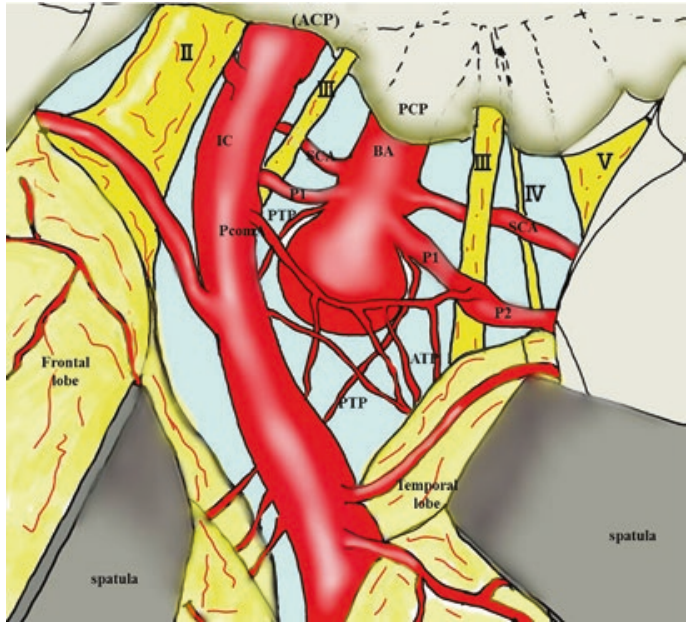

Fig. 17.1 Illustration of the basilar artery (BA) complex with BA tip aneurysm via the anterolateral approach. Note the anterior thalamoperforating arteries from the posterior communicating artery (p-com) and the posterior thalamoperforating arteries from the posterior cerebral artery (P1 and P2). II optic nerve, III oculomotor nerve, $I V$ trochlear nerve, $V$ first branch of trigeminal nerve, $A C P$ anterior clinoid process(resected), $P C P$ posterior clinoid process, $I C$ internal carotid artery, $S C A$ superior cerebellar artery anterior petrosal approach should be considered for aneurysms located more than $5 \mathrm{~mm}$ below the clinoid line.

\subsubsection{Approach}

\subsubsection{Extradural Temporopolar Approach ETA}

The pterional transsylvian approach and subtemporal transtentorial approach are usually used to treat upper BA aneurysms, but both have advantages and disadvantages. The anterior temporal approach, which retracts the temporal tip posteriorly, combines the advantages of both these approaches and facilitates the surgical trajectory from the anterolateral direction. The anterior temporal approach is a pure intradural approach based on dissecting the superficial middle vein and anterior temporal artery from the temporal lobe. The ETA is an epidural approach involving posterior displacement of the temporal lobe with
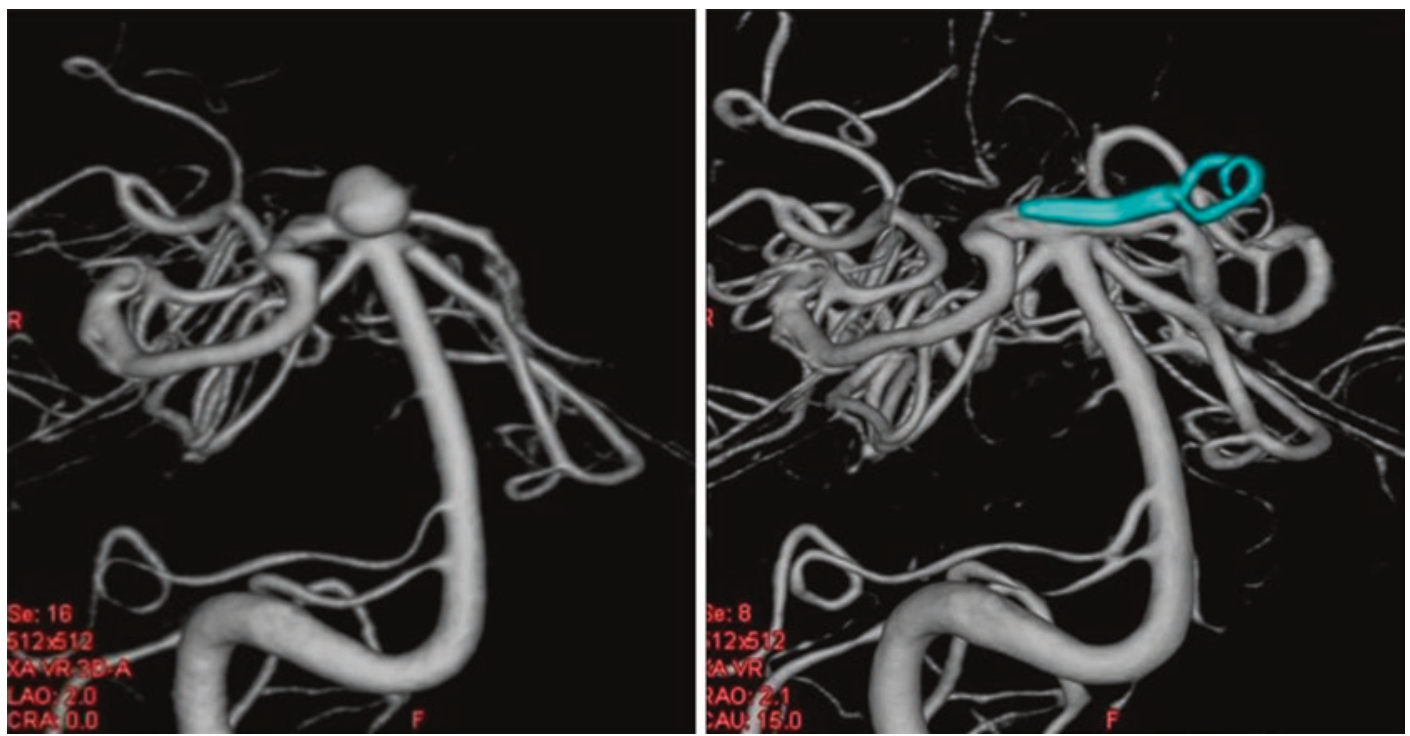

Fig. 17.2 Three-dimensional computed tomography angiograms of a case of basilar tip aneurysm. Left preoperative, Right postoperative 
the dura mater [2, 3] and includes extradural anterior clinoidectomy, optic canal opening, peeling the lateral wall of the cavernous sinus, and retraction of the temporal lobe over the dura mater. The ETA enables the neurosurgeon to pass through the open anterior part of the cavernous sinus to the retrocarotid space and interpeduncular cistern. Such epidural temporal lobe retraction is less likely to cause contusion. Furthermore, opening the oculomotor foramen and drilling the PCP provide space for securing the proximal BA. The use of the ETA for treatment of BA top aneurysm is discussed below.

\subsubsection{Patient Position, Skin Incision, and Craniotomy}

After induction of general anesthesia, lumbar spinal drainage is placed for cerebrospinal fluid (CSF) aspiration to relax the dural tension during the epidural procedures. The patient is placed in the supine position, and the head is rotated away from the operative side at about $30^{\circ}$ (Fig. 17.3). Semi-coronal skin incision and interfascial dissection of the temporal muscle fascia are then performed. The temporal muscle is detached from the superior temporal line but not incised. The temporal muscle is dissected subperiosteally

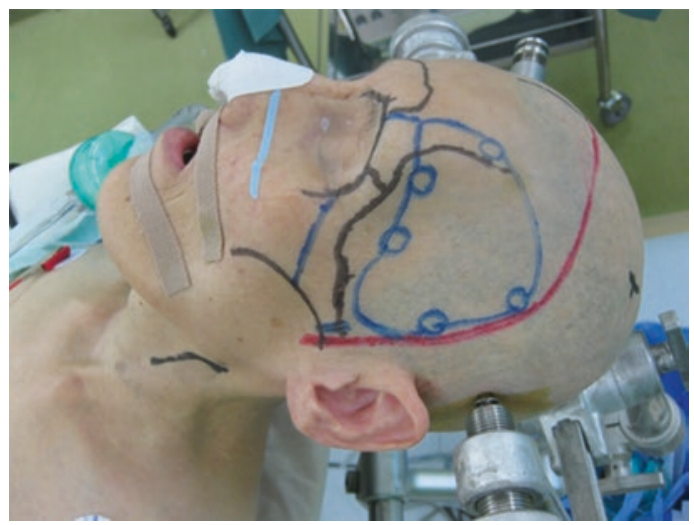

Fig. 17.3 Scheduled skin incision, frontotemporal craniotomy with orbitozygomatic osteotomy

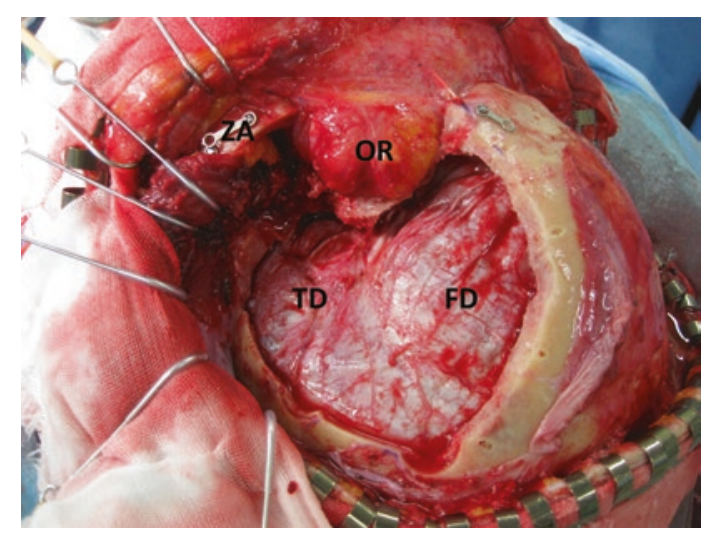

Fig. 17.4 After frontotemporal craniotomy with orbitozygomatic osteotomy. ZA zygomatic arch, $O R$ orbit (opened), $F D$ frontal dura mater, $T D$ temporal dura mater

and retracted posteroinferiorly using fish hooks. Standard frontotemporal craniotomy is performed with the anterior border at the midpoint of the orbit, and the temporal squama is rongeured out until the floor of the middle cranial fossa is exposed. If the orbitozygomatic approach is needed for specific cases such as high position BA tip aneurysm, the orbitozygomatic bar is removed as in the two-piece method (Fig. 17.4).

\subsection{Steps of the Surgery}

The frontal dura and the temporal dura are dissected subperiosteally until flush with the frontal and middle fossae. The lesser wing of the sphenoid and the orbital apex is drilled away until the meningo-orbital band is exposed. Dissection of the middle fossa dura is continued until the orifices of the superior orbital fissure (SOF) and foramen rotundum are exposed (Fig. 17.5). The roof of the SOF is opened to a width of $2 \mathrm{~mm}$ to expose the junction between the dura propria of the temporal lobe and the periosteal dura (Fig. 17.5 arrowhead). The meningo-orbital band is pulled proximally using forceps or a 


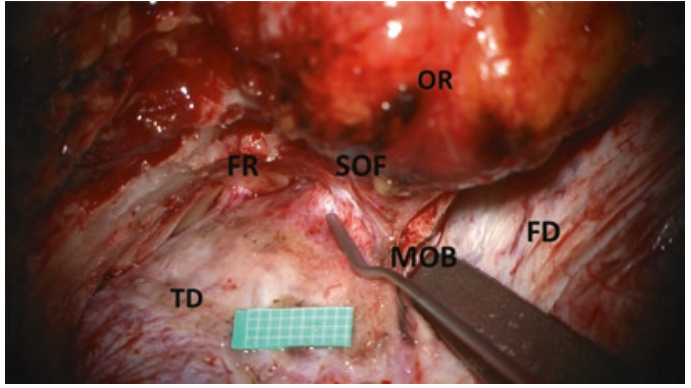

Fig. 17.5 Junction between the dura propria of the temporal dura mater (TD) and periosteum at the superior orbital fissure (SOF). $F D$ frontal dura mater, $M O B$ meningo-orbital band, $O R$ orbit. Arrowhead indicates the junction

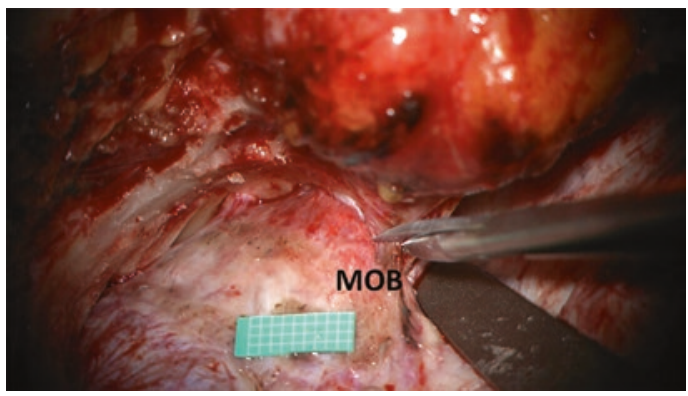

Fig. 17.6 Cutting the meningo-orbital band (MOB)

stitch and cut with micro-scissors for about $4 \mathrm{~mm}$. The tip of the micro-scissors should be pointed toward the exposed junction at the SOF (Fig. 17.6). Any bleeding from the meningoorbital artery should be coagulated and divided. Peeling of the dura propria from the inner membrane can be started anywhere, with the easiest point between the SOF and foramen rotundum, using a micro-dissector with a sharp edge. If the dura propria and inner membrane are tightly attached, sharp dissection using micro-scissors is recommended. The peeling is continued until the entire ACP is exposed epidurally. The trochlear nerve and first and second branches of the trigeminal nerve can be seen though the semitransparent inner membrane (Fig. 17.7). Care should be taken to maintain the sphenoparietal sinus at the dura propria side and to stop the peeling at the point where the sphenoparietal sinus drains into the cavernous sinus to prevent problems with venous congestion. Drilling of the ACP with a high-speed drill using saline irriga-

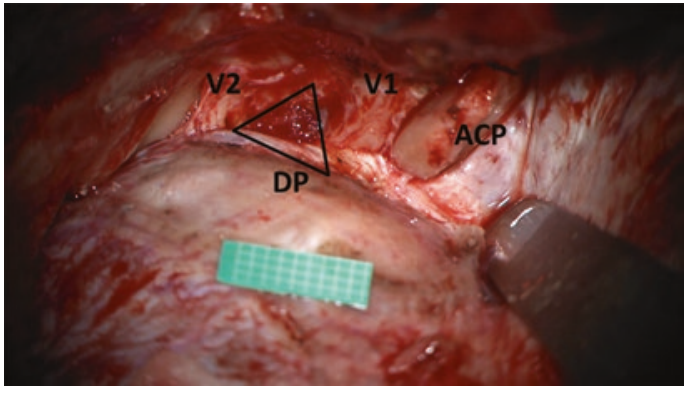

Fig. 17.7 After peeling of the dura propria (DP) of the temporal dura mater and exposure epidurally of the anterior clinoid process (ACP). V1 ophthalmic branch of the trigeminal nerve, $V 2$ maxillary branch of the trigeminal nerve. Open triangle shows Mullan's anterolateral triangle

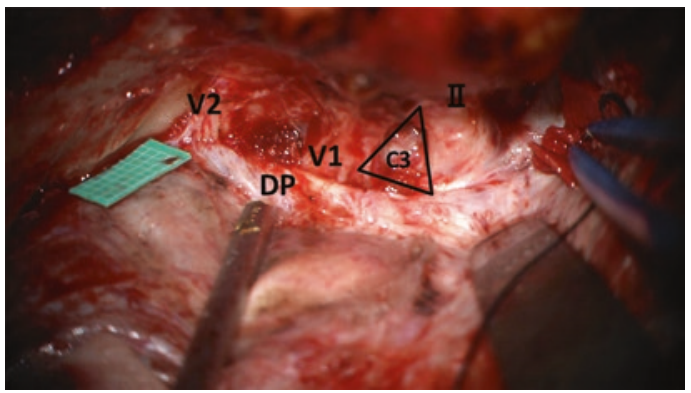

Fig. 17.8 Completion of epidural procedures after removal of the anterior clinoid process and opening of the optic canal. II optic nerve, III oculomotor nerve, VI ophthalmic branch of the trigeminal nerve, $V 2$ maxillary branch of the trigeminal nerve, $C 3$ clinoid segment of the internal carotid artery, $D P$ dura propria. Open triangle shows Dolenc's anteromedial triangle

tion is started from the lateral part of the ACP, and the optic canal is then opened partially in the medial part of the ACP. The ACP is hollowed like an egg shell, and the remainder of the ACP can be removed en bloc. The anterior loop of the ICA (clinoid segment, C3) in the opened clinoid space can be seen through the thin carotid oculomotor membrane. The partially opened optic canal can be enlarged using a micro-punch, and the remainder of the optic strut between the opened clinoid space and optic canal can be removed with either a small diamond drill or a micro-punch (Fig. 17.8). During the skull base drilling, if the posterior ethmoid sinus is opened and the mucous membrane is injured, a muscle piece should be inserted and sealed with fibrin glue to avoid CSF leakage. At this point, the tem- 
poral lobe with the temporal dura mater can be displaced posteriorly by $25-30 \mathrm{~mm}$ from the tip of the middle cranial fossa. The dura mater is cut along the sylvian fissure as far as the optic nerve, the falciform ligament is cut, and then the dural cutting is continued to the inferior frontal dura mater in an L-shaped fashion over the distal dural ring. Incision of the falciform ligament and distal dural ring will facilitate movement of the optic nerve and ICA. Before tentorial incision, the sylvian fissure should be opened widely, and the arachnoid membrane around the oculomotor nerve should be incised to free the nerve from the medial temporal lobe. By observing at the cisternal and extradural parts of the oculomotor nerve, the medial tentorial edge is cut for separation from the cavernous sinus around the oculomotor nerve. Following these procedures, the temporal lobe can be retracted posteriorly with the temporal dura mater to complete the ETA with the surgical corridor of the opened anterior part of the middle fossa and the cavernous sinus (Fig. 17.9). Opening of the oculomotor foramen facilitates mobilization of the oculomotor nerve. If the target pathology is located beneath the PCP, the latter can be drilled away to expose the pontine cistern to secure the proximal BA. The ICA is retracted medially to expose the BA complex with the BA tip aneurysm (Figs. 17.10 and 17.11). After temporary clips are applied to the

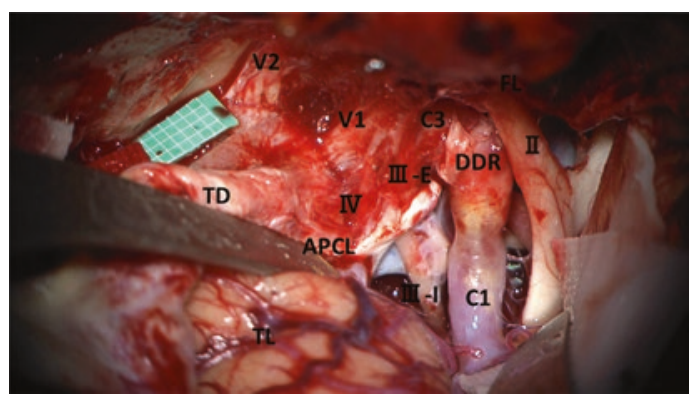

Fig. 17.9 After dural opening and tentorial incision. The falciform ligament (FL) and the distal dural ring (DDR) are incised. The tentorium is incised along the anterior petroclinoid ligament (APCL). The temporal dura is retracted posteriorly with the temporal dura mater (TD). II optic nerve, III-I intradural oculomotor nerve, III-E extradural oculomotor nerve, $I V$ trochlear nerve, $V 1$ ophthalmic branch of the trigeminal nerve, $V 2$ maxillary branch of the trigeminal nerve, $C 3$ clinoid segment of the internal carotid artery

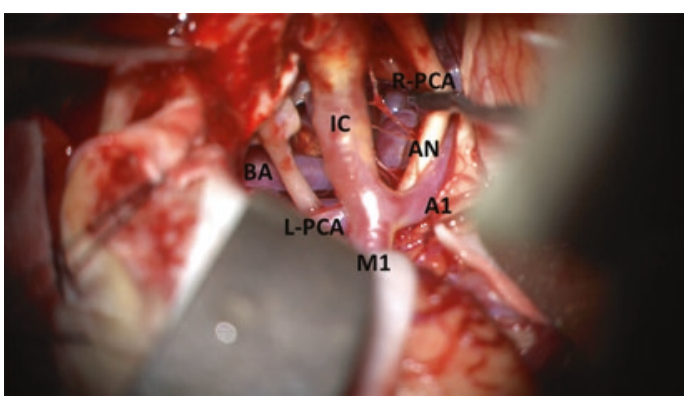

Fig. 17.10 Basilar artery (BA) complex and BA tip aneurysm. Note the small thalamoperforating arteries around the aneurysm (AN). IC internal carotid artery, $A 1$ anterior cerebral artery, $M 1$ middle cerebral artery, $R-P C A$ right posterior cerebral artery, $L-P C A$ left posterior cerebral artery

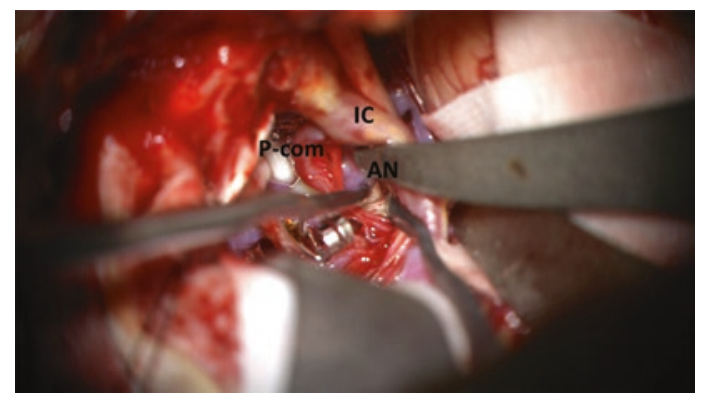

Fig. 17.11 Dissection of the basilar tip aneurysm (AN) via the retrocarotid space. $p$-com posterior communicating artery, $I C$ internal carotid artery

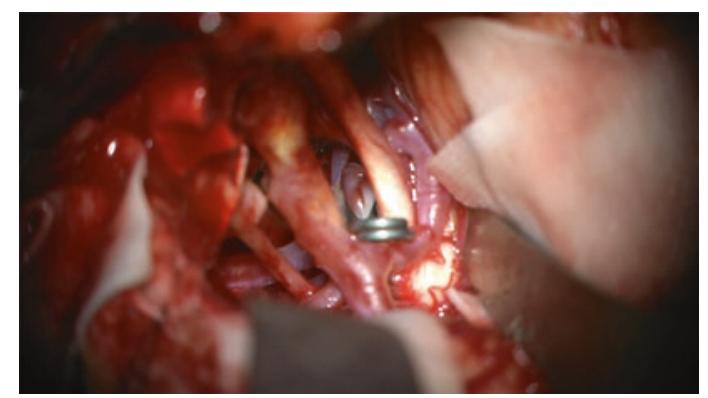

Fig. 17.12 After neck clipping of the basilar tip aneurysm

BA and bilateral posterior cerebral arteries, aneurysm dissection is started. If the posterior communicating artery obstructs the operative view, this vessel can be ligated and divided except for the fetal type. Great care is required to dissect the PTPAs in the vicinity of the aneurysm before neck clipping is performed (Fig. 17.12). 
After clipping of the aneurysm, the dural defect around the dural ring can be approximated with a small piece of harvested temporal fascia and fibrin glue. The skull is fixed with titanium plates, a subcutaneous drain is placed, and the wound is closed as usual.

\subsection{Specific Plans to Handle Complications and Expert Opinion}

\subsubsection{How to Avoid Heat Injury During Skull Base Drilling}

Constant cooling with saline irrigation is extremely important to avoid heat injury to the neurovascular structures. Before drilling the ACP, the ACP should be carefully dissected from the surrounding tissue because the oculomotor nerve passes along the inferolateral face of the ACP. The optic canal is partially opened with a drill and
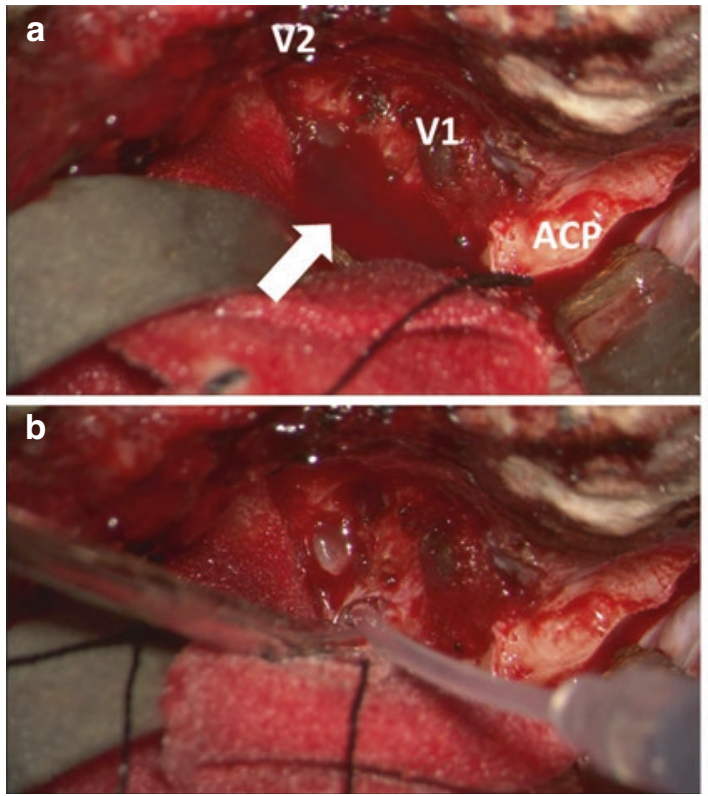

Fig. 17.13 How to control bleeding from the cavernous sinus. (a) Bleeding from Mullan's anterolateral triangle (arrow). (b) Direct injection of fibrin glue into Mullan's triangle. (c) Bleeding from Dolenc's anteromedial triangle (arrow). (d) Application of Surgicel ${ }^{\circledR}$ soaked with should be enlarged using a micro-punch. Opening the optic canal using a micro-punch can avoid heat injury.

\subsubsection{How to Control Bleeding from the Cavernous Sinus}

There are two major points of bleeding from the cavernous sinus, Dolenc's anteromedial triangle and Mullan's anterolateral triangle. Bleeding from the cavernous sinus can be controlled by packing with Surgicel ${ }^{\circledR}$ cotton soaked with fibrin glue or direct injection of fibrin glue into the sinus (Fig. 17.13).

\subsection{Important Observations and Postoperative Care}

CSF drainage (about $150 \mathrm{~cm}^{3} /$ day) from the lumbar spinal tube should be continued for several
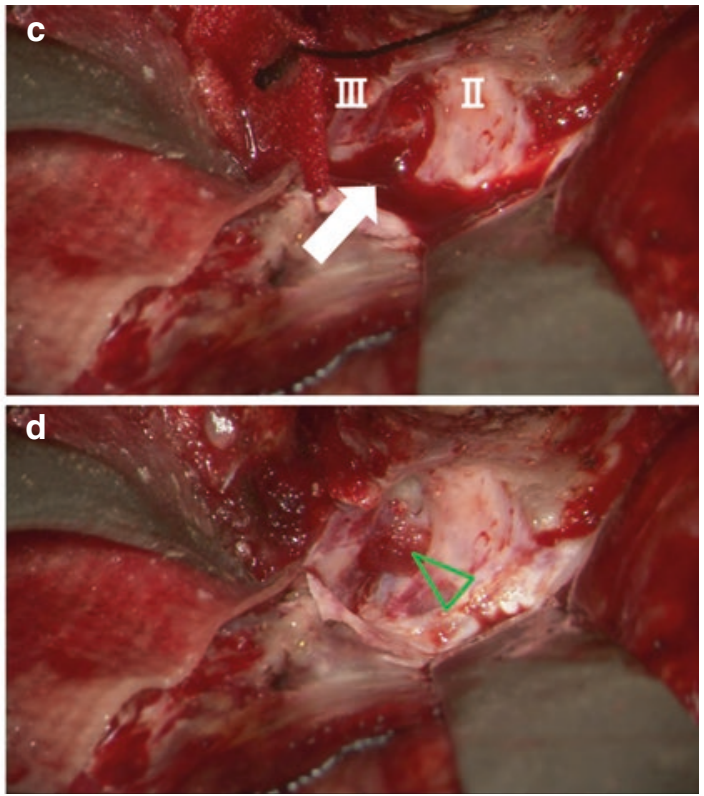

fibrin glue (open triangle). ACP anterior clinoid process, II optic nerve, III oculomotor nerve, VI ophthalmic branch of the trigeminal nerve, $V 2$ maxillary branch of the trigeminal nerve 
days (3-7 days) after the operation to prevent CSF rhinorrhea, especially if the ethmoid sinus is opened during surgery.

\section{References}

1. Park SQ, Bae HG, Yoon SM, Shim JJ, Yun IG, Choi SK. Morphological characteristics of the thalamoperforating arteries. J Korean Neurosurg Soc. 2010;47:36-41.
2. Day JD, Giannotta SL, Fukushima T. Extradural temporopolar approach to lesions of the upper basilar artery and infrachiasmatic region. J Neurosurg. 1994;81:230-5.

3. Day JD, Fukushima T, Giannotta SL. Cranial base approaches to posterior circulation aneurysms. J Neurosurg. 1997;87:544-54.

Open Access This chapter is licensed under the terms of the Creative Commons Attribution 4.0 International License (http://creativecommons.org/licenses/by/4.0/), which permits use, sharing, adaptation, distribution and reproduction in any medium or format, as long as you give appropriate credit to the original author(s) and the source, provide a link to the Creative Commons license and indicate if changes were made.

The images or other third party material in this chapter are included in the chapter's Creative Commons license, unless indicated otherwise in a credit line to the material. If material is not included in the chapter's Creative Commons license and your intended use is not permitted by statutory regulation or exceeds the permitted use, you will need to obtain permission directly from the copyright holder. 